\title{
Model-based Analysis of Decentralized Fluidic Systems in Machine Tools
}

\author{
Linart Shabi*, Juliane Weber, and Jürgen Weber \\ Institute of Fluid Power, TU Dresden, Helmholtzstraße 7a, 01069 Dresden, Germany \\ E-mail: linart.shabi@tu-dresden.de,juliane.weber@tu-dresden.de,mailbox@ifd.mw.tu-dresden.de \\ * Institute of Fluid Power, TU Dresden, Helmholtzstraße 7a, 01069 Dresden, Germany
}

\begin{abstract}
Power losses in machine tools, e.g. during production process, are converted into thermal energy. This leads to a warming of the machine frame and further machine parts and accordingly to the displacement of the tool center point (TCP) of the machine. Consequently, the accuracy of the machine during the production process is reduced. The warmed-up parts or components need to be cooled; therefore, fluidic systems are installed to prevent this effect. Previous research projects mainly focused on the energy demand of the machine tool and its main drives, reducing the energy consumption by developing more efficient components and control strategies. However, the thermal behavior of the fluidic systems, especially of the cooling system, has not yet been described in detail. Therefore, a detailed analysis of the existing cooling system and its effectivity is necessary.
\end{abstract}

The main target of this paper is to analyze the thermal behavior and improve the system structure of the cooling system in a demonstration machine tool. This investigation will help to examine the efficiency of the cooling system for an idle and a manufacturing process. This makes it possible to study new concepts for the system structure of the cooling system in order to ensure a uniform temperature distribution of the machine tool at minimal energy consumption.

Firstly, the paper will describe the main fluidic systems of the demonstration machine DBF630 with a special focus on the cooling system. Secondly, with the aid of experimental investigation the thermal behavior of the cooling system will be investigated. Furthermore, a network-based model of the cooling system for two processes is developed and validated against the measured data. Lastly, the new concept of a decentralized supply unit will be studied with the network-based simulation model.

It can be shown that the decentralization of the cooling system has a high potential towards a better thermal behavior and a lower energy consumption of the machine tool. The simulation results show a more stable temperature profile of the components as well as a lower energy consumption of the cooling system.

Keywords: Fluidic system, Machine tools, Simulation, Cooling system, Thermo-elastic deformation, Energy consumption

\section{Introduction}

Besides increasing the productivity, the demand for a high accuracy of the workpieces and improved energy efficiency of the production processes also increases. The thermo-elastic characteristics of machine tools are strongly controlled by the fluidic systems such as cooling system. Typical fluidic systems in machine tools are the cooling system, the cooling lubricant system, the lubrication system, and the hydraulic system. On the one hand, fluidic systems (cooling, cooling lubricant and lubrication system) allow heat dissipation from the overall machine tool structure as well as the main components in order to achieve lower temperature changes and to homogenize the temperature distribution. On the other hand, fluidic systems themselves are essential energy consumers and, therefore, a significant heat source within the machine tool. Previous studies were carried out in $[1,2,3]$ to analyze and determine the energy consumption and the energy distribution at the machine tools. However, these focus on decreasing the energy consumption of the components of the machine tools through constructive measures as well as developing a shutdown control of components during waiting times. The thermal behavior and the effectivity of the fluidic systems, especially the cooling system has not yet been described in detail. Therefore, a detailed analysis of the existing cooling system structures and their thermal behavior is necessary to increase the efficiency of the machine tools and provide a uniform temperature distribution. A well-designed fluidic system should therefore 
improve the accuracy while at the same time being more energy-efficient.

The main goal of this work is to analyze the thermal behavior of the cooling system and its subsystems for two different processes, idle and manufacturing process. This investigation will help to obtain information concerning the efficiency of the cooling system. This makes it possible to derive new optimization approaches, such as a decentralization of the system structure. This allows to improve the thermal behavior of the cooling system by providing a stable temperature profile of the components and reducing the energy consumption of the cooling system.

Chapter 2 gives an overview of the fluidic system with the special focus on the cooling system. Here, the status quo analysis of the cooling system for an idle and a manufacturing process as well as the modeling approach for the simulation are shown. In chapter 3 the decentralization concept of the system structure and its potential against the current system structure is presented.

\section{Status quo analysis of the fluidic system}

The selected and experimentally analyzed demonstrator machine in Figure 1, type Scharmann DBF630, has three linear feed axes with ball screw drive $(\mathrm{X}, \mathrm{Y}, \mathrm{Z})$ and one rotary feed axis (B). The additional $U$ axis is used only for the turning process and is inactive during the drilling or milling processes. The spindle of DBF630 is a gear spindle with two gear steps. In gear step 1, the spindle rotation speed ranges to $1200 \mathrm{~min}-1$, and the transmission ration is $i 1=8.2080$. In this step, a high cutting performance due to high torque of $1700 \mathrm{Nm}$ could be applied. In gear step 2, the spindle rotation speed ranges to $3500 \mathrm{~min}-1$, the transmission ration is $\mathrm{i} 2=1.5$. So the spindle speed range can be varied by the transmission ratio and by motor through the frequency converter. The overall driving power of the machine is $35 \mathrm{~kW}$ [4].

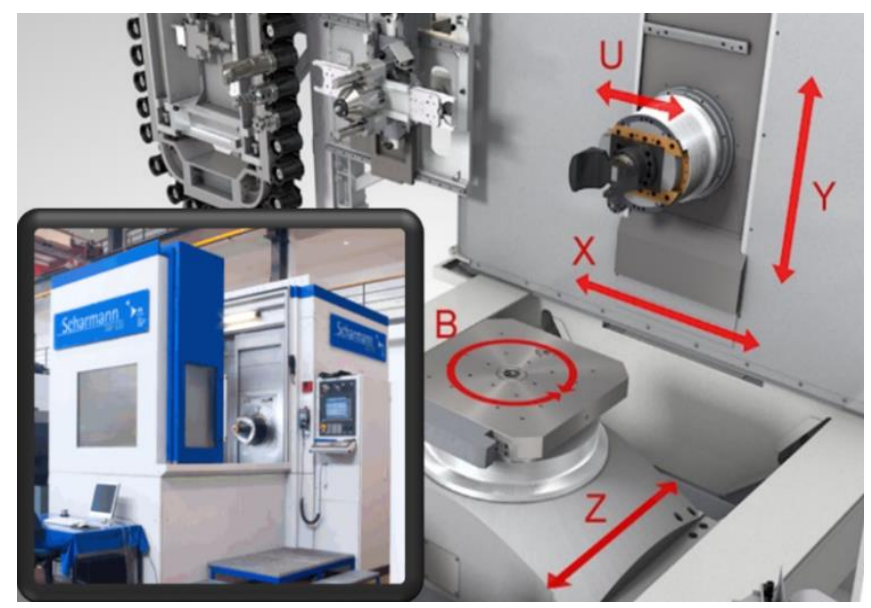

Figure 1: Schematic representation of axes configuration of DBF630 [6]

Detailed information about the demonstration machine and the function of their fluidic systems can be found in [5, 6]. As mentioned previously, the fluidic system in machine tools are divided into cooling, hydraulic, lubrication and cooling lubricant system. Figure 2 shows the main fluidic systems of the demonstration machine DBF630.

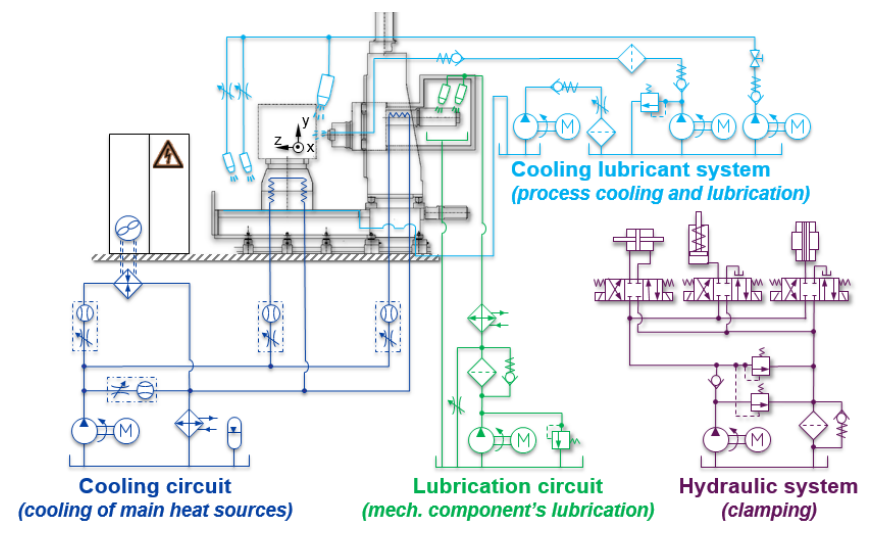

Figure 2: Main fluidic system of DBF630 [5]

\subsection{Status quo analysis of the cooling system}

As shown in Figure 3, the cooling system consists of three subsystems: electrical cabinet 6 , rotary table 4 , and main drive 7. A fixed displacement pump 14 supplies the cooling medium with a flow rate of $401 / \mathrm{min}$ at a pressure level of 5.2 bar according to the machine documentation. As cooling medium, a mixture of water and at least $20 \%$ Antifrogen $₫$ is used. The rotary table 4 and the main drive 7 are cooled directly by the cooling medium that flows through integrated cooling channels. The cooling of the electrical cabinet takes place via an air heat exchanger 12. The cooling unit 13 in DBF630 is integrated directly into the return flow side and cools down the heated fluid to a set temperature. Its functional principle is a two-point temperature control similar to that of refrigerators. The cooling unit is switched on as soon as the temperature exceeds the upper limit of the set temperature, and is switched off when the cooling medium is cooled to the lower set temperature.

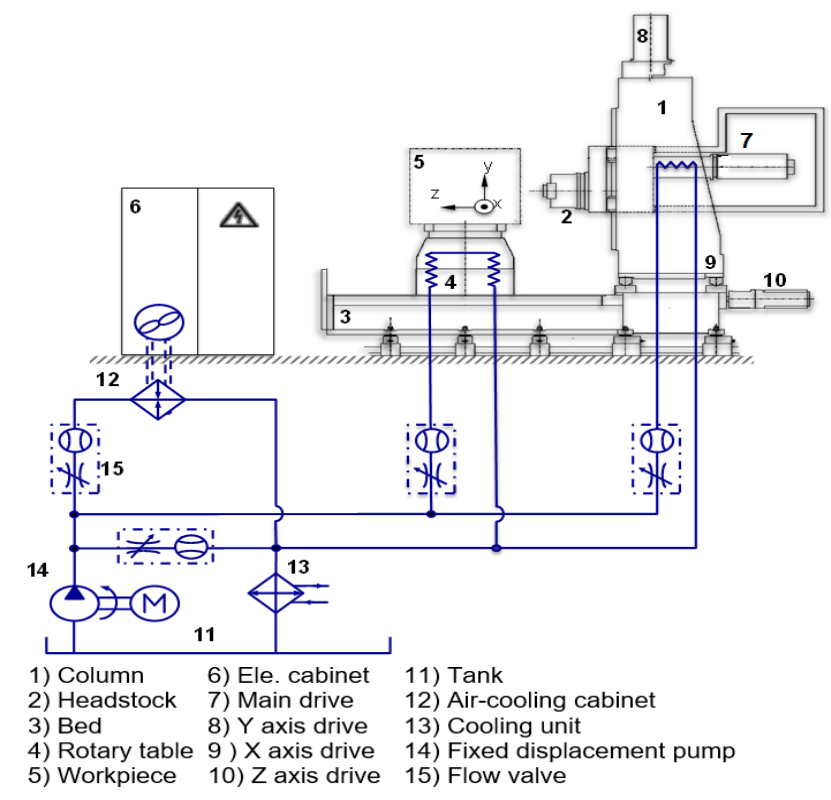

Figure 3: Cooling system of DBF630 
In order to determine the thermal behavior as well as the energy consumption of the machine and of the individual subsystems, experimental machine measurements were carried out. Additional temperature sensors (thermocouples type T class 1) and flow sensors were installed. For the investigation of DBF630, four phases were chosen: warm up process, idle process, setup process, and manufacturing process. The results of the idle and the manufacturing process are shown in this paper. The idle process of the DBF630, see Figure 4, is based on the ISO 230-3 [7], where typical load cycles are considered. Referring to the idle process of DBF630 in Figure 4, at the beginning, the main drive accelerates several times to its maximum speed of $3500 \mathrm{~min}^{-1}$ and decelerates again. Afterwards, the rotary table rotates with its maximum rotational speed for approx. $10 \mathrm{~s}$. Following this, the linear axes $\mathrm{X}, \mathrm{Y}$ and $\mathrm{Z}$ are moved.

In the production process, several steel blocks (S235 JR) were face-milled with two different tools. The tool-specific characteristics and the resulting cutting conditions are shown in Figure 5.

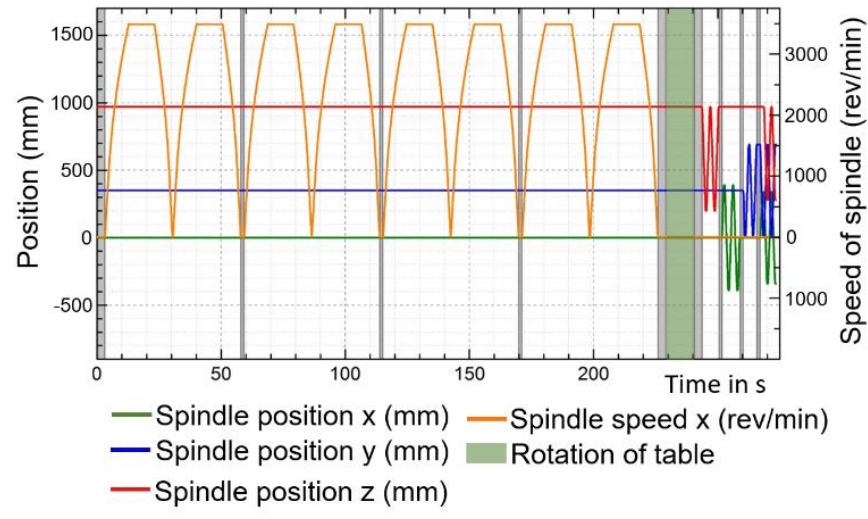

Figure 4: Idle process of DBF [4]

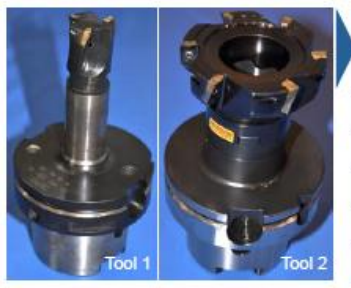

\begin{tabular}{|lll}
\hline \multicolumn{1}{|c}{} & $\begin{array}{l}\text { Tool 1 } \\
\text { R390-32T16-11M }\end{array}$ & $\begin{array}{l}\text { Tool 2 } \\
\text { R390-080Q27-17M }\end{array}$ \\
\hline Cutter diameter & $32 \mathrm{~mm}$ & $63 \mathrm{~mm}$ \\
\hline Cutting depth & $2 \mathrm{~mm}$ & $4 \mathrm{~mm}$ \\
\hline Speed & $2100 \mathrm{~min}^{-1}$ & $1100 \mathrm{~min}^{-1}$ \\
\hline Feed rate & $630 \mathrm{~mm} / \mathrm{min}$ & $660 \mathrm{~mm} / \mathrm{min}$ \\
\hline Teeth number & 3 & 6 \\
\hline Coolant supply & Internal, external & External \\
\hline
\end{tabular}

Figure 5: Cutting tools used for manufacturing process [4]

Due to the two-point temperature control of the cooling unit, all temperature profiles of the idle process can be divided into three phases: in phase 1 and 3, the cooling unit is off whereas it is being activated in phase 2. Figure 6 shows the temperature of the cooling medium at the inlet side and the outlet side of the electrical cabinet, the rotary table, the main drive and tank. At the electrical cabinet and the rotary table, the temperature difference between the outlet and the inlet is about $-1 \mathrm{~K}$, so the cooling medium is cooled while the components are warmed up. Only the main drive is cooled actively during all phases. Here, the outlet temperature of the cooling medium is higher $(\Delta \mathrm{T}=1.5 \mathrm{~K})$ than the inlet temperature.
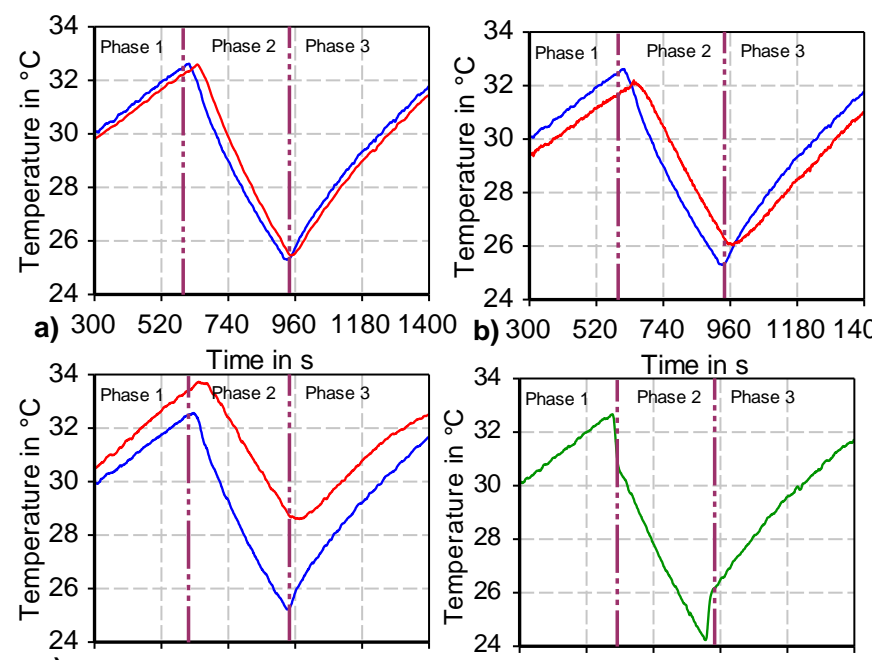

b) 30052074096011801400

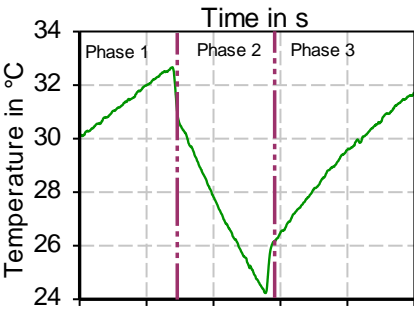

c) $300 \quad 520 \quad 740 \quad 960 \quad 1180 \quad 1400$ d) $300 \quad 520 \quad 740 \quad 960 \quad 1180 \quad 1400$ Time in $\mathrm{s}$

- Inlet temperature Time in s

- Tank temperature

Figure 6: Temperature characteristics in the idle process a) Electrical cabinet b) Rotary table c) Main drive d) Tank

With the aid of the measured temperatures $\mathrm{T}$ and volume flow $\dot{m}$; the heat flow $\dot{Q}$ and the thermal energy $E$ of the electrical cabinet, the rotary table and the main drive (spindle) are calculated as follows:

$$
\begin{gathered}
\dot{Q}=P_{\text {th }}=\dot{m} \cdot C_{\text {fluid }} \cdot \Delta T+\frac{1}{\rho} \cdot \Delta P \\
E=\int_{t 1}^{t 2} P_{t h} d t
\end{gathered}
$$

Figure 7 and Table 1 depict the heat flow and the thermal energy in the idle process. In phases 1 and 3, the generated heat of the spindle is dissipated by the cooling medium. However, the electrical cabinet and the rotary table are heated by the cooling medium. A clear difference is recognized when the cooling unit is switched on in phase 2 between $t_{o n}=600 \mathrm{~s}$ and $\mathrm{t}_{\mathrm{off}}=900 \mathrm{~s}$.
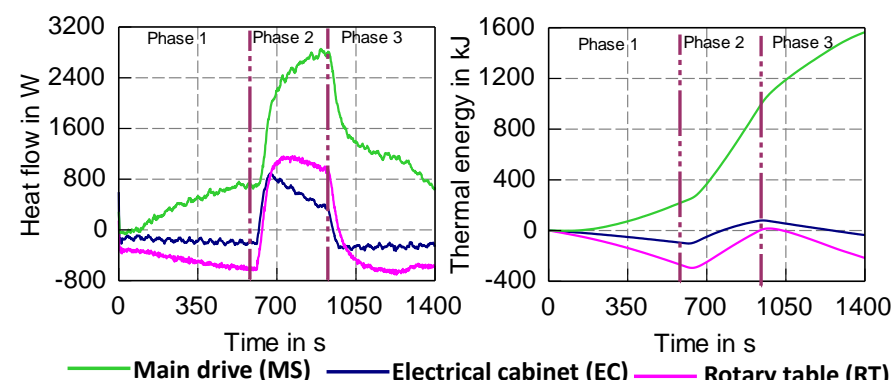

Figure 7: Heat flow and thermal energy in the idle process

When the cooling liquid is actively cooled, the heat flow increases and all three components transfer thermal energy to the cooling system and are thus cooled. 
Table 1: Thermal energy of electrical cabinet $(E C)$, rotary table (RT) and main drive (MS) during idle process

\begin{tabular}{|l|l|l|l|}
\hline & $\Delta Q_{E C}$ in $k J$ & $\Delta Q_{R T}$ in $k J$ & $\Delta Q_{M S}$ in $k J$ \\
\hline Phase 1 & -99 & -280 & 230 \\
\hline Phase 2 & 165 & 248 & 656 \\
\hline Phase 3 & -101 & -183 & 651 \\
\hline
\end{tabular}

These three different cooling phases can also be found in the temperature profile of the manufacturing process, see Figure 8. In phases 1 and 3 , the cooling unit is activated while in phase 2 it is turned off. The temperature development in the electrical cabinet and rotary table is similar to that in the idle process. The temperature difference between the outlet and the inlet at the electrical cabinet and the rotary table for phase 2 is about $-0.3 \mathrm{~K}$ to $-1 \mathrm{~K}$, so the cooling medium is cooled while the components are warmed up. Only the main drive is cooled actively during all phases, the maximum temperature difference between outlet and inlet is about $3.3 \mathrm{~K}$.

With the aid of equation (1) and (2), the thermal energy of electrical cabinet, rotary table and main drive for the manufacturing process are calculated. The cooling unit is switched on in phase 1 (from $0 \mathrm{~s}$ to $95 \mathrm{~s}$ ) and in phase 3 (from $820 \mathrm{~s}$ to $1100 \mathrm{~s}$ ). Figure 9 and Table 2 give an overview concerning the heat input during the manufacturing process.
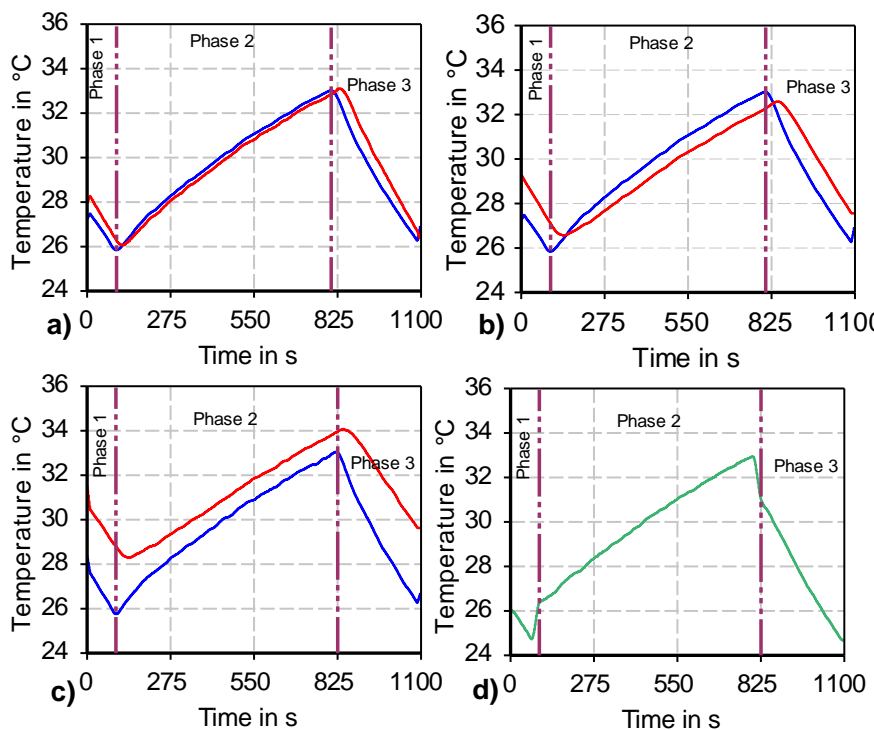

- Inlet temperature

Outlet temperature

- Tank temperature

Figure 8: Temperature characteristics in the manufacturing process a) Electrical cabinet b) Rotary table c) Main drive d) Tank

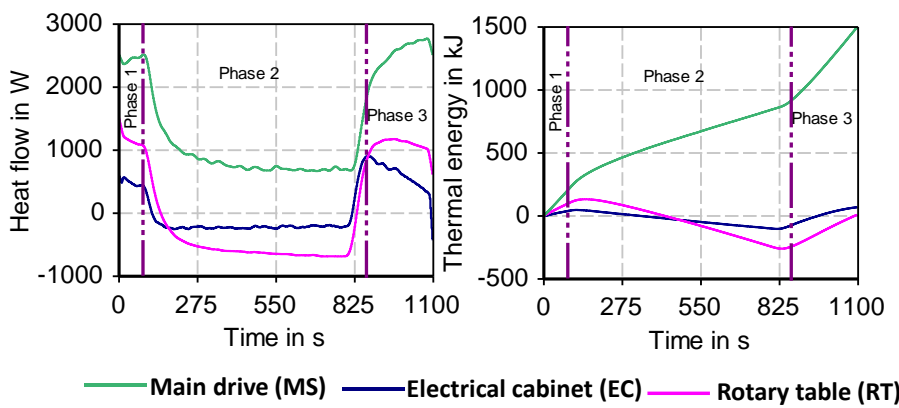

Figure 9: Heat flow and thermal energy in the manufacturing process

Table 2: Thermal energy of electrical cabinet $(E C)$, rotary table (RT) and main drive (MS) during manufacturing process

\begin{tabular}{|l|l|l|l|}
\hline & $\Delta Q_{E C}$ in $k J$ & $\Delta Q_{R T}$ in $k J$ & $\Delta Q_{M S}$ in $k J$ \\
\hline Phase 1 & 44 & 110 & 233 \\
\hline Phase 2 & -58 & -147 & 627 \\
\hline Phase 3 & -32 & -248 & 642 \\
\hline
\end{tabular}

\subsection{Model development and simulation}

Simulation models offer a good and flexible way to investigate the behavior of a system. In order to be able to derive reliable statements from simulation models, it is necessary to analyze the behavior of the real system. The aim of the modelling is to describe the real system with appropriate mathematical and physical approaches [8]. The rules of Kirchhoff's circuit laws, Kirchhoff's node rule, and Kirchhoff's loop rule in electrical engineering and the laws for series and parallel connection of resistance can be applied to the hydraulic and thermodynamic domain. The calculation of the thermal and hydraulic resistances $R_{t h}$ and $R_{\text {hy }}$ between two nodes is based on the following relationships [9, tab. 1]:

$$
\begin{aligned}
& R_{t h}=\frac{\Delta T}{\dot{Q}} \\
& R_{h y}=\frac{\Delta p}{\dot{V}}
\end{aligned}
$$

Regarding to node rule of Kirchhoff:

$$
\begin{aligned}
& T=\frac{1}{C_{t h}} \int \sum_{i=1}^{n} \dot{Q}_{l} \cdot d t \\
& p=\frac{1}{C_{h y}} \int \sum_{i=1}^{n} \dot{V}_{l} \cdot d t
\end{aligned}
$$

Figure 10 illustrates a basic thermo-hydraulic network model. The fluid volume or the mass properties are concentrated in the junction points. These nodes display the hydraulic and thermal capacities. Thermal and hydraulic resistors connect the nodes to each other. 


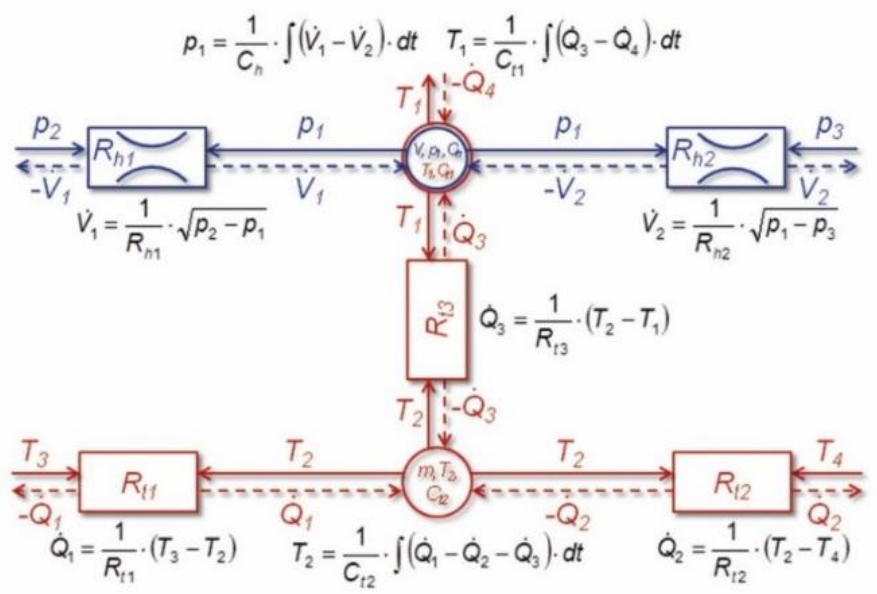

Figure 10: Basic description of the thermo-hydraulic network model [10]

Additionally, the convective heat transport through the cooling medium in the pipes is taken into account. The heat transfer coefficients are determined by the following equations [11]:

$$
\begin{gathered}
R e=\frac{D_{H} \cdot \dot{V}}{v \cdot A} \\
\operatorname{Pr}=\frac{C_{\text {fluid }} \cdot \rho \cdot v}{\lambda_{\text {fluid }}} \\
N u=0.0235 \cdot\left(R e^{0.8}-230\right) \cdot\left(1.8 \cdot \operatorname{Pr}^{0.3}\right. \\
-0.8) \cdot\left[1+\left(\frac{d_{i}}{l}\right)^{\frac{2}{3}}\right] \\
\alpha_{\text {con. }}=\frac{\lambda_{\text {fluid }} \cdot \mathrm{Nu}}{d_{i}}
\end{gathered}
$$

A Simulation model of the cooling system was developed based on the model description as well as the machine documents. The cooling system consists mainly of a pump, flow control valves, hydraulic pipes, a cooling unit and the component (electrical cabinet, rotary table, and main drive) as heat source. In the simulation, each hydraulic pipe is modelled by a hydraulic volume and a hydraulic resistance. The geometrical parameters (length, inner and outer diameter) of the pipes are taken directly from the machine documentation. Moreover, the heat transfer between the pipes and the environment is taken into account. Figure 11 shows the model structure of the cooling system implemented in the simulation.

For the model-based study of the system, a domain-crossing system simulation with SimulationX (ESI ITI GmbH) was carried out. Figure 12 and Figure 13 show a direct comparison of the simulation and the measurement of the idle and the manufacturing process. The simulation model of the cooling system shows a high accuracy of the thermal and hydraulic quantities of the considered components. The simulation model could therefore be validated and will be used for further studies such as a sensitivity analyses [12].

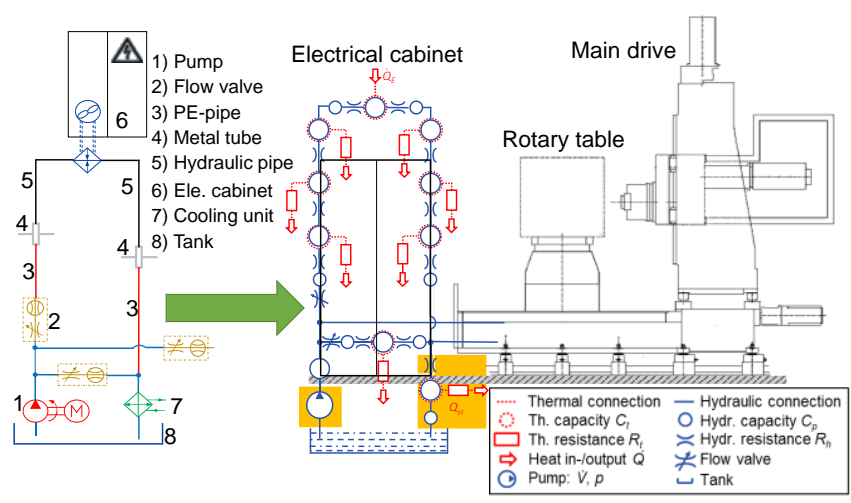

Figure 11: Model structure of cooling system for the idle and manufacturing process
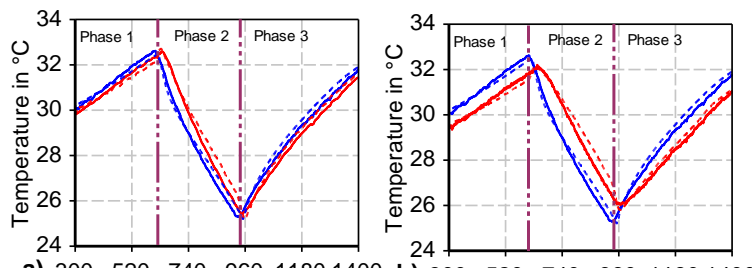

a) $300 \quad 520740 \quad 96011801400$ b) $300 \quad 520 \quad 740 \quad 960 \quad 11801400$
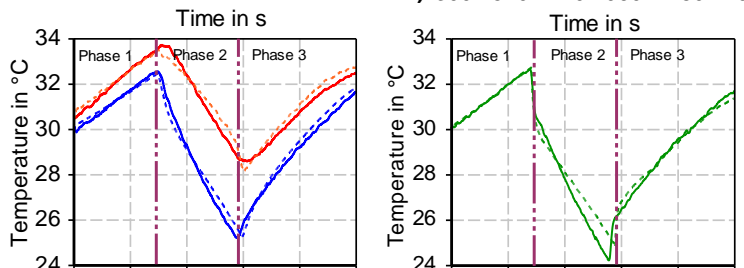

$\begin{array}{lllllllll}\text { c) } 300 \quad 520 \quad 740 \quad 960 \quad 11801400 & \text { d) } 300 \quad 520 \quad 740 & 960 & 1180 & 1400\end{array}$ Time in $\mathrm{s}$

Time in s

_ Inlet temperature measurement $\quad$ - - - Inlet temperature simulation

- Outlet temperature measurement - - -Outlet temperature simulation

_ Tank temperature measurement $\quad$ - - -Outlet temperature simulation

Figure 12: Comparison of temperature development simulation and measurement in the idle process a) Electrical cabinet b) Rotary table c) Main drive d) Tank
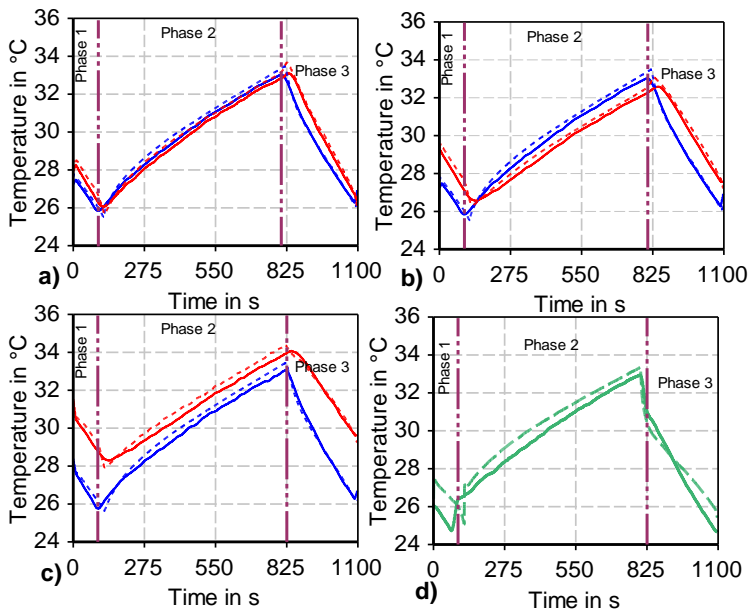

_Inlet temperature measurement _ - - Inlet temperature simulation

— Outlet temperature measurement - - - Outlet temperature simulation

_ Tank temperature measurement - - - Outlet temperature simulation

Figure 13: Comparison of temperature development simulation and measurement in the manufacturing process a) Electrical cabinet b) Rotary table c) Main drive d) Tank 


\section{Potential analysis of decentralized system structure}

The investigation of two demonstration machines shows that the fluidic systems together require in the idle process $44 \%$ (DBF630) and 51\% (DMU80) of the total energy consumption of the machine tool $[5,6]$. Therefore, there is a major potential for reducing the energy demand and increasing the efficiency of machine tools by improving the function of the fluidic systems. Furthermore, the investigation of the DBF630 in the idle and in the manufacturing process depicts that sufficient cooling capacity exists but that the cooling is insufficiently adjusted to the process and to the individual demand of the machine components. In order to address this deficit, it is necessary to think about new concepts and new structures of fluidic systems. Such an approach is the decentralization of the system structure. Figure 14 displays the decentralization of the cooling system at different individualization stages [13].
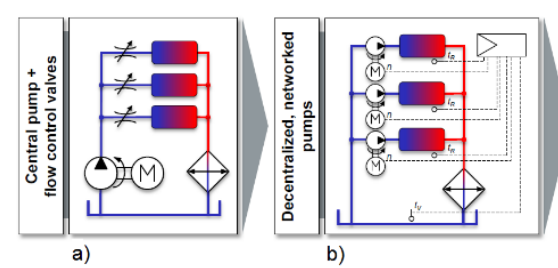

b)

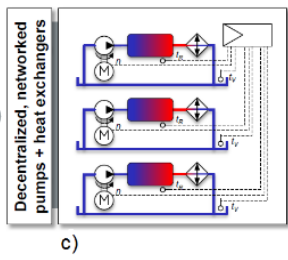

\section{Figure 14: Decentralization steps of system structure a) Current structure b) Distributed pump, stage I c) Distributed pump and cooling unit, stage II}

Decentralized, independent systems allow an adequate and component-specific supply and thus a targeted temperature control. The studied variants for optimizing the cooling system are evaluated according to the following criteria: stable temperature profile in the components, low pump pressure (short hydraulic pipes), and low energy requirements. As previously shown, in a centralized cooling system, a fixed displacement pump delivers a constant volume flow to the system. The volume flow does not correspond to the individual requirement and to the temperature development of the respective component. Therefore, the cooling medium is cooled while the components are warmed up (negative heat flow). Another deficit is the two-point temperature control of the cooling unit. This controls the mixing temperature in the tank and not the component temperature.

\section{Optimization according to stage I}

Figure 15 shows the first optimization step, stage I, applied to the DBF630. The components are cooled with individual pumps that are connected to a common tank and cooling unit. This system design does not require a flow control valve to control the volume flow to the components. The control of the cooling system compares the actual and set temperature of the individual components and on this basis, adjusts the speed-controlled pumps. Therefore, each pump delivers a different, demand-oriented cooling volume flow. If the temperature development in the component does not exceed predefined limits, the pump remains inactive. The cooling unit remains in the two-point temperature control and refers to the mixing temperature of all components. The temperature signal $\vartheta_{\text {actual }}$ corresponds to the average component temperature. The temperature detection is carried out via sensors in the components. For this purpose, a suitable concept for sensor integration is necessary.

In the decentralized simulation model, three set temperatures were defined for the components, for the electrical cabinet $\vartheta_{\text {set }}$ $=26{ }^{\circ} \mathrm{C}$, for the rotary table $\vartheta_{\text {set }}=27{ }^{\circ} \mathrm{C}$ and for the motor spindle $\vartheta_{\text {set }}=28{ }^{\circ} \mathrm{C}$. This individual temperature control is not possible with the current system structure. In addition, an average equivalent heat flow was determined from the calculated heat flows in the idle process for each individual component. It was $1500 \mathrm{~W}$ for the main drive and $150 \mathrm{~W}$ for the electrical cabinet and rotary table. Moreover, only static operating points of the cooling system were considered in the simulation, so that the thermal capacity of the components was not required.

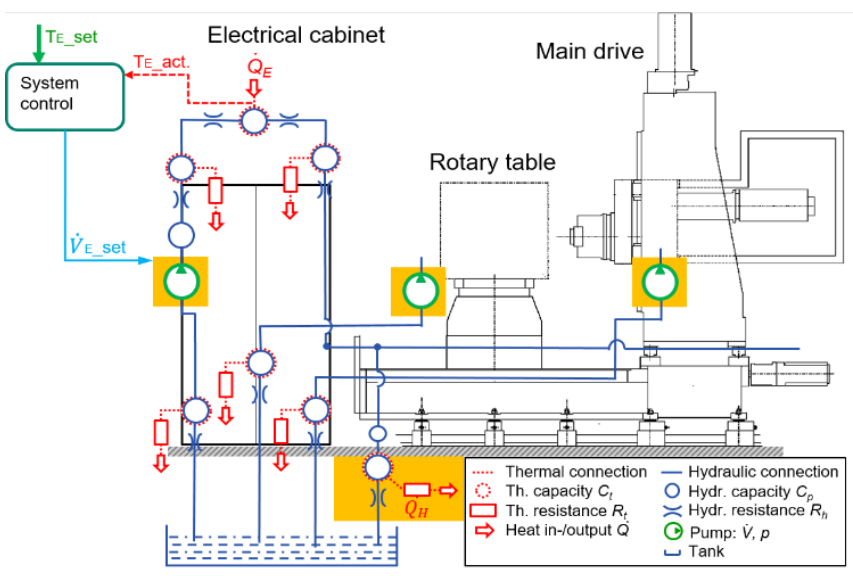

Figure 15: Decentralization of the cooling system structure according to stage I

Figure 16 shows the simulation results of the fluid outlet temperature development in the electrical cabinet, rotary table and main drive as well as the required cooling volume flow in the decentralized system in comparison of the central system. Figure 16 a - c represent the direct comparison of the fluid outlet temperature between central and decentralized system. The fluid outlet temperature of the component remains constant at the set temperature $\left(26^{\circ} \mathrm{C}, 27^{\circ} \mathrm{C}, 28^{\circ} \mathrm{C}\right)$ in the decentralized system despite an increased power loss. In the central system, the fluid outlet temperature increases with rising power losses, therefore the actual temperature of the fluid is dependent on the heat input to the component. With a closer look into the volume flow characteristics of the pumps, it should be noted that the cooling medium flow in the decentralized system increases with rising power losses, in contrast to the current system, were the volume flow is constant. In example of the machine tools main drive (motor spindle), the pump in the decentralized system delivers the cooling medium flow based on the components temperature, depending on the current power loss. The delivered flow rate varies between $8 \mathrm{l} / \mathrm{min}$ to $16 \mathrm{l} / \mathrm{min}$.

As shown in Figure 16 d, the volume flow control is a way to design the system in a more energy-efficient way based on each individual components demand. For the simulation, it is 
assumed that the pumps are located next to the components, i.e. shorter pipe lengths were taken into account. For this reason, the pump pressure is also very low.
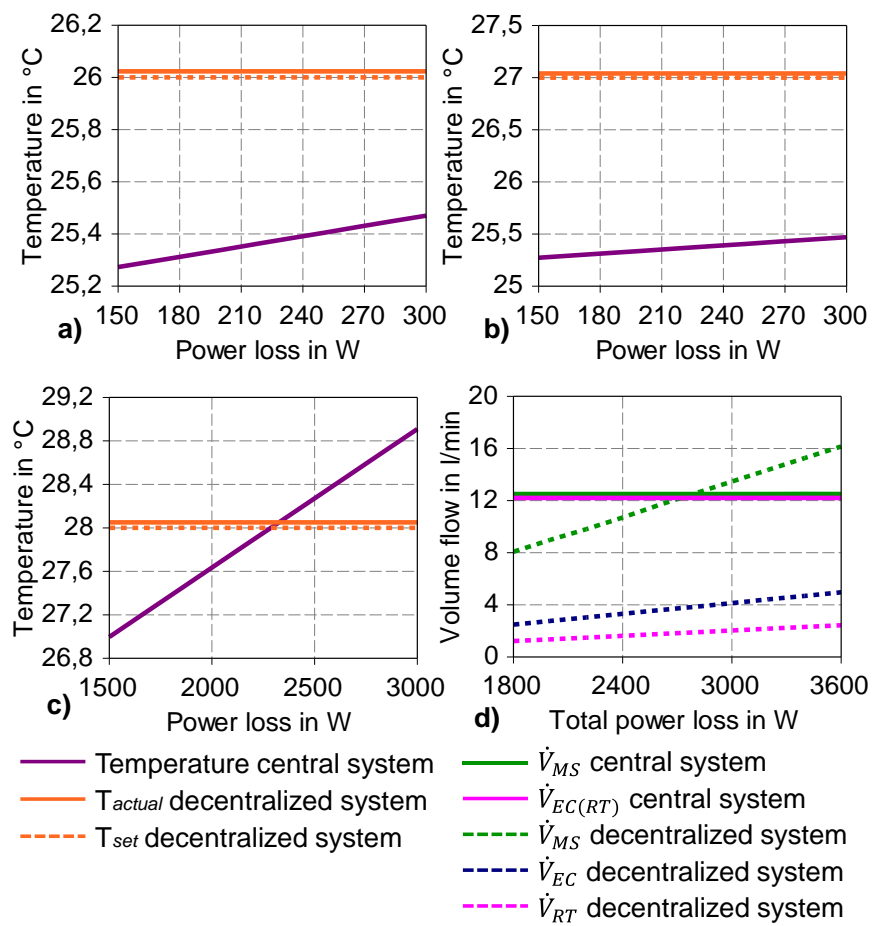

Figure 16: Simulation results fluid outlet temperature of component in the decentralized system in comparison of central system a) Electrical cabinet b) Rotary table c) Main drive d) volume flow profile

Figure 17 illustrates the total hydraulic power of the pumps in the central and decentralized system for different power losses. The total hydraulic power at maximal power loss of the pumps in the decentralized system is $80 \mathrm{~W}$. The hydraulic power of the fixed pump in the central system is approx. $370 \mathrm{~W}$ (40 1/min at $5.5 \mathrm{bar}$ ), hence, a significant energy saving is possible. The new functional principle of the pump is significantly more energy-efficient compared to the current state of the DBF630 with a continuous flow volume.

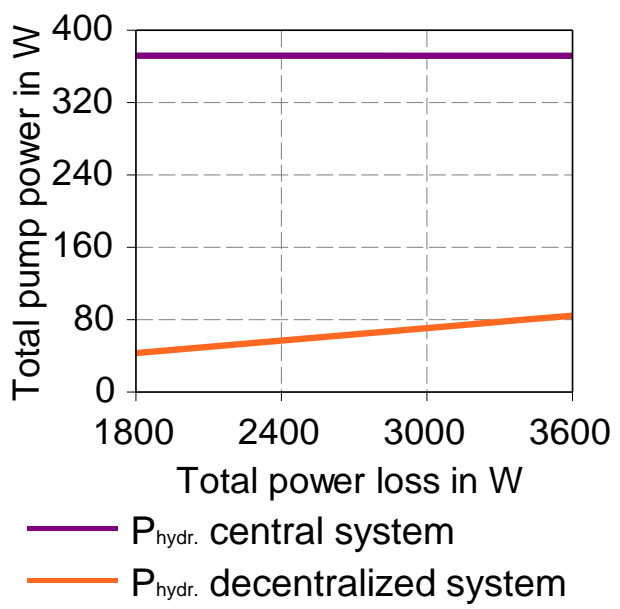

Figure 17: Pump performance, decentralization stage I

\section{Summary}

In order to increase the productivity and manufacturing accuracy of the workpieces with a simultaneous decrease of the energy consumption, the minimization of thermo-elastic deformation and, consequently, a minimization of the TCP displacement is necessary. The warmed-up parts or components need to be cooled, therefore, fluidic systems such as cooling system are an essential element for controlling the thermo-elastic characteristics of machine tools.

The main goal of the paper was to analyze the thermal behavior and improve the current system structure of the cooling system in a demonstrator machine tool.

Firstly, the authors showed the existing fluidic system of the demonstrator machine DBF630 and described in detail its cooling system. With aid of experimental investigation and machine documents a network-based model of the cooling system for two processes, the idle and manufacturing process, was developed and validated against the measured data. The validated model was used for further studies such as decentralization of supply unit.

As a result, the investigation of the cooling system of the DBF630 in the idle and in the manufacturing process shows that sufficient cooling capacity is available but that the cooling is insufficiently adjusted to the process and to the individual demand of the machine components (negative heat flow in Figure 7 and Figure 9). Furthermore, independent systems and demand-oriented component cooling was studied with the help of a simulation model. The delivered coolant flow is controlled individually according to the temperature development in the component. The simulation results have shown that a stable fluid outlet temperature of the components compared to the initial state can be achieved. Moreover, the demand-oriented fluid supply of the pumps leads to a successful improvement of the machine tools energy efficiency. This results in a reduction of the required total pump power for the DBF630 from $370 \mathrm{~W}$ to $80 \mathrm{~W}$.

\section{Acknowledgements}

The presented research activities are part of the project "Thermo-energetic description of fluid systems" (Ref. No. CRC/TR 96, A04). The authors would like to thank the German Research Foundation (DFG) for the financial support.

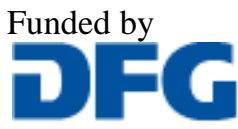




\section{Nomenclature}

\begin{tabular}{|c|c|c|}
\hline Designation & Denotation & Unit \\
\hline$A$ & Area of pipe & $\mathrm{m}^{2}$ \\
\hline$C_{\text {fluid }}$ & $\begin{array}{l}\text { Specific heat capacity at } \\
\text { constant pressure }\end{array}$ & $\mathrm{J} / \mathrm{kg} \cdot \mathrm{K}$ \\
\hline$C_{t h}$ & Thermal capacity & $\mathrm{J} / \mathrm{K}$ \\
\hline$C_{h y}$ & Hydraulic capacity & $\mathrm{m}^{3} / \mathrm{Pa}$ \\
\hline$D_{H}$ & Hydraulic diameter & $\mathrm{m}$ \\
\hline$d i$ & Inner diameter & $\mathrm{m}$ \\
\hline$E$ & Energy & $\mathrm{J}$ \\
\hline$l$ & Length of pipe & $\mathrm{m}$ \\
\hline$\dot{m}$ & Mass flow & $\mathrm{Kg} / \mathrm{s}$ \\
\hline $\mathrm{Nu}$ & Nusselt number & - \\
\hline $\operatorname{Re}$ & Reynolds number & - \\
\hline$p$ & Pressure & $\mathrm{Pa}$. \\
\hline$\Delta p$ & $\begin{array}{l}\text { Outlet/ Inlet pressure } \\
\text { difference }\end{array}$ & $\mathrm{Pa}$. \\
\hline $\operatorname{Pr}$ & Prandtl number & - \\
\hline$\dot{Q}, P_{t h}$ & Heat flow & $\mathrm{W}$ \\
\hline$t$ & Time & $\mathrm{s}$ \\
\hline$Q_{E C}, Q_{R T}, Q_{M S}$ & $\begin{array}{l}\text { Thermal energy of the } \\
\text { electrical cabinet, rotary table, } \\
\text { main drive }\end{array}$ & $\mathrm{J}$ \\
\hline$\dot{V}$ & Volume flow & $\mathrm{m}^{3} / \mathrm{s}$ \\
\hline$\vartheta$ & Temperature & ${ }^{\circ} \mathrm{C}$ \\
\hline$\alpha$ & Heat transfer coefficient & $\mathrm{W} / \mathrm{m}^{2} \cdot \mathrm{K}$ \\
\hline$T$ & Temperature & $\mathrm{K}$ \\
\hline$\Delta T$ & Outlet/ Inlet temp. difference & $\mathrm{K}$ \\
\hline$\lambda_{\text {fluid }}$ & Thermal conductivity & $\mathrm{W} / \mathrm{m} . \mathrm{K}$ \\
\hline$v$ & Kinematic viscosity & $\mathrm{m}^{2} / \mathrm{s}$ \\
\hline$\rho$ & Density & $\mathrm{Kg} / \mathrm{m}^{3}$ \\
\hline
\end{tabular}

\section{References}

[1] B. Denkena and T. Graber. NCplus Prozess- und wertschöpfungsorientiert gesteuerte Werkzeugmaschine. PZH Verlag, 2013

[2] C. Brecher. Effizienzsteigerung von Werkzeugmaschinen durch Optimierung der Technologien zum Komponentenbetrieb-EWOTeK. Apprimus Verlag, Aachen, 2012.

[3] U. Götze, HJ. Koriath, A. Kolesnikov, R. Lindner, J. Paetzold and C. Scheffler. Energetische Bilanzierung und Bewertung von Werkzeugmaschinen. Tagungsband
Energieeffiziente Produkt- und Prozessinnovationen in der Produktionstechnik eniPROD. Chemnitz, 2010.

[4] J. Weber and J. Weber. Thermo-Energetic Analysis of the Fluidic Cooling Systems in Tooling Machines. 9th International Fluid Power Conference, Aachen, March 24-26, 2014.

[5] J. Weber and J. Weber. Thermo-energetic Modelling of Fluid Power Systems. In: Großmann K, editor. Thermoenergetic Design of Machine Tools. Heidelberg Springer-Verlag; 2015. p. 49-60.

[6] L. Shabi, J. Weber and J. Weber. Analysis of the Energy Consumption of Fluidic Systems in Machine Tools. The 50th CIRP Conference on Manufacturing Systems, Taiwan, May 3th - 5th, 2017

[7] International standard ISO 230-3. Test code for machine tools, part 3: Determination of thermal effects. Second edition 2007-08-15.

[8] D. Abel and A. Bollig. Rapid Control PrototypingMethoden und Anwendungen. Springer Verlag Berlin Heidelberg 2006. s. 77-80

[9] J. Weber and J. Weber. Thermo-energetic analysis and simulation of the fluidic cooling system of motorized high-speed spindles. SICFP2013, Linköping, Sweden June 3th-5th ,2013

[10]J. Weber and J. Weber. Analyse und Simulation der fluidischen Kühlung einer einfach gewendelten Motorspindelkühlhülse. O+P Journal 3/2013

[11]E.Doering, H.Schedwill and M. Delhi. Grundlagen der Thermodynamik.7 Auflage, Springer Verlag Vieweg 2012

[12]L. Shabi, J. Weber and J. Weber. Investigation of Fluidic Systems in Machine Tools. 22nd International Conference on Hydraulics, Pneumatics, Tools, Sealing Elements, Fine Mechanics, Specific Electronic Equipment \& Mechatronics (HERVEX 2016), November 9-11, Romania, 2016

[13]. Weber and J. Weber. Thermo-Energetic Analysis of the Fluidic Systems in Cutting Ma-chines. 10th International Fluid Power Conference, Dresden, March 08-10, 2016. 